\title{
Biometric Alterations of Mouse Embryonic Eye Structures Due to Short-Term Folic Acid Deficiency
}

\author{
Ouafa Sijilmassi $\mathbb{1}^{\mathrm{a}, \mathrm{b}}$, José Manuel López-Alonso ${ }^{\mathrm{b}}$, Aurora Del Río Sevilla ${ }^{\mathrm{a}}$, Jorge Murillo González \\ and María del Carmen Barrio Asensio ${ }^{a}$
}

aFaculty of Optics and Optometry, Anatomy and Human Embryology Department, Universidad Complutense De Madrid, Madrid, Spain; ${ }^{b}$ Faculty of Optics and Optometry, Optics Department, Universidad Complutense De Madrid, Madrid, Spain; 'Faculty of medicine, Anatomy and Human Embryology Department, Universidad Complutense De Madrid, Madrid, Spain

\section{ABSTRACT}

Purpose: Folic acid (FA) is an essential nutrient for normal embryonic development. FA deficiency (FAD) in maternal diet increases the risk of several defects among the progeny, especially, neural tube defects. The eye begins its development from the neural tube; however, the relationship between FAD and ocular development in the offspring has been little explored and it isn't known how the FAD affects the formation of the eye. Our objective was to analyze the effect of maternal FAD on mouse embryos ocular biometry.

\section{Introduction}

Methods: Female mice $\mathrm{C} 57 / \mathrm{BL} / 6 \mathrm{~J}$ were distributed into three different groups, according to the assigned diet: control group fed a standard FA diet (2 mg FA/ $\mathrm{kg}$ ), FAD group for short term fed (0 mg $\mathrm{FA} / \mathrm{kg}+1 \%$ succinylsulfathiazole) from the day after mating until day 14.5 of gestation, and FAD group for long term fed the same FA-deficient diet for 6 weeks prior mating and continued with this diet during gestation. A total of 57 embryos (19 embryos of each dietary group) at 14.5 gestational days were evaluated. As indicators of changes in ocular biometry, we analyze two parameters: area and circularity of the lens and whole eye, and the area of the retina. The program used in the treatment and selection of the areas of interest was ImageJ. The statistical analysis was performed by IBM SPSS Statistics 19. Results: Regarding the measures of the area, FA-deficient lenses and eyes were smaller than that of controls. We have also observed increase in the size of the neural retina, spatially, in embryos from females fed FAD diet during long term. On the other hand, as regard to circularity measures, we have seen that eyes and lenses were more circular than control.

Conclusion: Maternal FAD diet for a very short term generates morphological changes in ocular structures to the offspring.
Eye morphogenesis in vertebrates begins in the early stages of development. The first appearance of the optic primordium in the human embryo begins forming on about day 22 as bilateral evaginations of the neuroectoderm of the forebrain (prosencephalon) which still remains open. ${ }^{1}$ In the mouse it appears at about the 9th day of gestation. ${ }^{2}$ These evaginations, optic vesicles, continue to proliferate laterally and gradually approach the surface ectoderm as the forebrain closes. Finally, the distal portion of the vesicle makes contact with the overlying surface which induces the formation of lens primordia, the lens placodes. In a next step, coordinated invagination of the optic vesicle and the lens placode and results in the formation of a double-layered optic cup and the lens vesicle and provide the first indication of the final shape of the eye. The inner layer of the optic cup forms the neural retina, while the outer layer of the optic cup gives rise to the retinal pigment epithelium. ${ }^{3}$

The eye structure is derived from different embryological origins. Ocular surface tissues involve the differentiation of ectoderm
ARTICLE HISTORY

Received 21 May 2018 Revised 26 October 2018

Accepted 1 November 2018

\section{KEYWORDS}

Folic acid deficiency; ocular biometry; eye abnormalities; development; statistical analysis cells to form corneal and conjunctival epithelium, lens, lacrimal, and tarsal glands. Migration and differentiation of periocular mesenchymal cells, which predominately originate from the neural crest, give rise to sclera, choroid, corneal endothelium, vitreous, and the stroma of the cornea, iris, and ciliary body. Nonetheless, neuroectodermal cells form the retina, the iris, and the ciliary body epithelium. ${ }^{2,3}$

Folates are essential vitamins for the development of the nervous system and craniofacial structures. ${ }^{4,5}$ Low maternal folic acid (FA) status has been associated with increased risk of neural tube defects as a result of incomplete development of the central nervous system. ${ }^{6,7}$ This tube closes around the 28 th day of gestation, ${ }^{8}$ often before a woman knows she is pregnant. Since deficiency of FA causes different alterations in the neural tube and it is known that the eye develops around the time of closure of the neural tube, ${ }^{2}$ it is reasonable that eye malformations are likely in situations of folate deficiency. Even knowing the importance of this vitamin there have been few studies related to ocular development and FA deficiency, nevertheless, how FA interferes in the development of

CONTACT Ouafa Sijilmassi o.sijilmassi@ucm.es Faculty of Optics and Optometry, Anatomy and Human Embryology Department, Universidad Complutense De Madrid, Arcos de Jalón, 118, Madrid 28037, Spain

Color versions of one or more of the figures in the article can be found online at www.tandfonline.com/icey.

(c) 2018 Taylor \& Francis Group, LLC 
ocular structures has not been much explored and it seems that the appearance of severe ocular malformations is linked

70 to a drastic reduction of FA in mother's diet. ${ }^{9,10}$ On the other hand, it has not been able to establish whether congenital eye defects such as anophthalmia and microphthalmia could be reduced by the effect of FA in pregnant women. ${ }^{11,12}$

In a previous study, ${ }^{13}$ we have found that FA deficiency 75 alters the expression of two extracellular matrix molecules, collagen IV and laminia-1, in embryonic mouse lens. Because of the fundamental role of the extracellular matrix in embryonic development, ${ }^{14}$ we suspect that abnormal basement membranes could alter the morphology of some eye elements. 80 Ocular biometric changes are not visualized macroscopically neither microscopically in short-time period of FA deficiency. In this research, we performed these experiments to identify if some ocular biometric parameters are affected basing us in microscopic images of immunohistochemical staining that delimits the structures to be measured. For this, we are going to study the biometry of the lens, retina, and entire eye, studying the area and circularity of the previous eye structures. In order to assess changes in the area and circularity of some structures due to deficiency of FA, we carried

90 out this experiment by means of image processing techniques using ImageJ and Matlab. ${ }^{15,16}$

\section{Materials and methods}

The animal facilities and the experimental protocol used in the studies were reviewed and ethically approved by the

95 Animal Experimentation Committee of the Universidad Complutense of Madrid. Mice were kept in the animal house of the School of Medicine at the Universidad Complutense of Madrid.

\section{Animals and diets}

100 Eight-week-old C57/BL/6J female mice (Harlan Laboratories, Barcelona, Spain) were housed at a constant temperature of $22 \pm 2^{\circ} \mathrm{C}$ and $12 / 12 \mathrm{~h} \mathrm{light/dark} \mathrm{cycles.} \mathrm{They} \mathrm{had} \mathrm{free} \mathrm{access} \mathrm{to}$ diet and water.

Female mice were distributed into three different groups according to the diet assigned:

Control group, 10 females fed standard diet containing $2 \mathrm{mg} \mathrm{FA} / \mathrm{kg}$. Deficit group D2, 12 females fed for 2 weeks (short term) FA deficiency (FAD) diet $(0 \mathrm{mg} / \mathrm{kg}$ diet $+1 \%$ succinylsulfathiazole) from the day after mating until day 14.5

110 of gestation. Deficit group D8, 10 females fed for 8 weeks (long term) FAD diet: 6 weeks prior pregnancy and continued with this diet during 2 weeks of gestation. Both diets were purchased from Harlan Laboratories, Inc., Indianapolis, IN, USA.

115 Diets administrates were based on the experimental dietary findings ${ }^{17}$ and in accordance with the nutritional requirements for mice, ${ }^{18}$ modifying only the FA content, $2 \mathrm{mg} \mathrm{FA} / \mathrm{kg}$ diet (control diet) or $0 \mathrm{mg} \mathrm{FA} / \mathrm{kg}$ diet (FAD diet). The addition of $1 \%$ succinylsulfathiazole in FAD diet inhibits the bacterial synthesis of folate in the intestine and causes a severe FA deficiency. ${ }^{19,20}$ This diet is usual in folate deficiency studies. We have previously found that FAD diet administered to female mice modifies liver folate levels and is also timedependent. Folate levels in livers were halved after 2 weeks on the FAD and reduced about 6 -fold after 8 weeks. ${ }^{21}$

\section{Immunohistochemistry staining protocol}

Females were anesthetized with carbon monoxide and sacrificed by cervical dislocation at day 14.5 (E14.5) equivalent to $\sim 7$ weeks of human gestation. ${ }^{22}$ The embryos were removed by cesarean section and decapitated. A total of 57 embryos (19 embryos of each dietary group) were collected. Embryos placed in cold sterile phosphate-buffered saline and fixed for $48 \mathrm{~h}$ in buffered formaldehyde. After fixing, heads were covered in paraffin. Frontal sections were made with a thickness of $5 \mu \mathrm{m}$ and placed on slides.

Sections were incubated for $2 \mathrm{~h}$ at room temperature with either 1:200 polyclonal rabbit IgG anti-mouse laminin (SigmaAldrich) or 1:200 polyclonal rabbit IgG anti-human type IV collagen (ICN Biomedical Inc., Aurora, OH, USA).

Labeling was developed using the Rabbit/Mouse EnVision ${ }^{\mathrm{TM}}$ Peroxidase System, a peroxidase-conjugated dextran polymer (Dako Corp., Carpinteria, CA, USA), and 3,30diaminobenzidine (DAB kit) as the chromogen (Dako Corp.). ${ }^{23}$ All slides were analyzed with a Leica DMRB microscope and subsequently photographed using a Leica DFC 320 digital camera with $\times 10$ magnification and a pixel size of 3.45 $\times 3.45 \mu \mathrm{m}^{2}$.

\section{Performing biometric measures}

As detailed earlier, the images that we discuss were photographed with a Leica camera attached to a Leica DMRB microscope with magnification of $\times 10$. The program used in the treatment of these images was ImageJ: it enabled us to select a particular area of the image directly, so we could select areas of interest, which are irregular or cannot be easily captured by automatic procedures.

Once these areas were selected, the program calculated various parameters. These included the area, measured in units of pixels (area), and circularity (Circ.) (see Figure 1). Area is defined by ImageJ as the number of pixels inside the selected region of interest (ROI). Circularity of the ROI is defined as

$$
\text { Circ }=4 \pi \cdot\left(\frac{\text { Area }}{{\text { (Perimeter })^{2}}^{2}}\right)
$$

And ranges from 0 (infinitely elongated polygon) to 1 (perfect circle).

Since the ROI is selected by the observer, there may be doubt about whether the error made by the observer in the selection is larger or smaller than the dispersion among mice of the same sample group (control, D2, D8). A test was performed with the lens in the control group because it is the structure that apparently shows less variability between mice. To perform the test, the measurements of area and circularity of the lens were repeated N-times for each of the mice in our control group $(n=19)$. Then, the standard deviation of the results for each mouse was calculated. This 

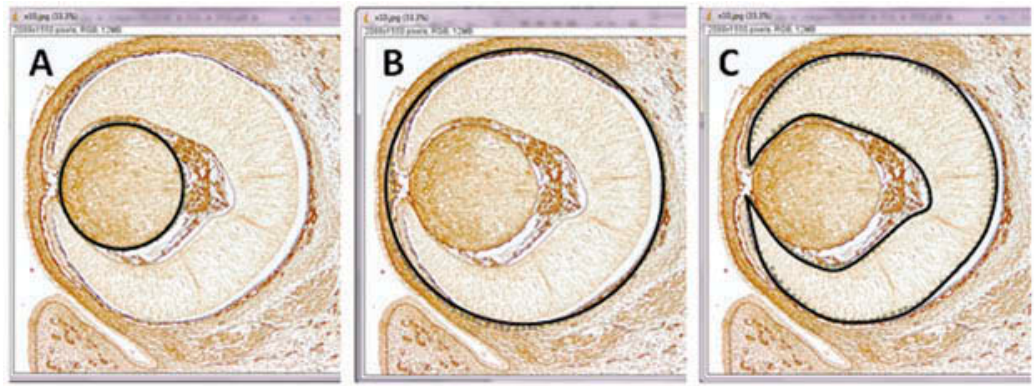

Figure 1. Region of interest (ROI) of area and circularity measurements: a) lens, b) entire eye, and c) retina are selected. Frontal section of E14.5 mouse embryo with $\times 10$ magnification labeled with anti-type IV collagen.

value was taken as an uncertainty of the measurements associated with the observer selection of the ROI. After that, the standard deviation of the mean values for each mouse in the control group was calculated. This last value was taken as the uncertainty for the whole group. Mean area of the lens, its standard deviation, and the mean observer error (for the control group) were 285653,45 562, and 1340 pixels, respectively.

It was observed that the standard deviation for the whole group of the mean area of the lens (45 563 pixels) was higher than the average error made by the observer (1340 pixels). From these data, we ruled out the observer error in the other groups, D2 and D8. Only a single measure was then taken for each mouse, because the variability between mice was much greater than the observer error. In the case of measures of circularity, the error of the observer cannot be ignored and is added to these measures.

A first analysis was performed using IBM SPSS Statistics 19 (SPSS 19) ${ }^{24}$ which calculated a series of statistical parameters (mean, standard deviation, etc.). To have a finer analysis of the results, we will study the standard deviation of the data, but also higher-order statistics such as skewness and kurtosis. A second analysis was carried out using MATLAB to estimate the previous parameters (mean, standard deviation, skewness, and kurtosis) using bootstrap techniques. ${ }^{25}$ Bootstrap is a resampling technique used to estimate the probability distribution of various estimates of a population as well as its confidence interval. In our case, we apply them to obtain the probability distribution of the estimator of the mean, standard deviation, skewness, and kurtosis. A total of 100 bootstrap replicas have been used. Normally this number is sufficient to obtain adequate confidence intervals. ${ }^{26}$ Subsequently, we used the Kruskal-Wallis statistical test to test the hypothesis that the probability distributions of the mean, standard deviation, skewness, and kurtosis are different between the control and D2 and D8 groups. The level of significance chosen is $p<0.05$. With this method we can compare if the differences in these estimators are significant or not using the population of each group (control, D2, and D8).

In order to further explore the changes in these parameters, we make use of the Generalized Extreme Value distribution (GEV) as a fitting family to fit the data of each group to a probability distribution. The GEV distribution is a family of continuous probability distributions established within the extreme value (EV) theory, which is a branch of statistics that studies the deviations of the mean of the probability distributions. The GEV distribution unites three families of distributions: the Gumbel, Fréchet, and Weibull distributions into a unique family to permit a continuous range of possible shapes. It is often used as an approximation to model the maxima of long sequences of random variables. We used this distribution because it is applicable to 'abnormal' data (malformations as in our case). The class of GEV distributions is very flexible with the tail shape parameter $\xi$ controlling the shape and size of the tails of the three different families of distributions subsumed under it. These three families of distributions can be nested into a single parametric representation. ${ }^{27-29}$ This representation is known as the GEV distribution and is given by Equation (2):

$$
G(z)=\exp \left\{-\left[1+\xi\left(\frac{z-\mu}{\sigma}\right)\right]^{\frac{-1}{\xi}}\right\}
$$

where $\mu$ is the location parameter, $\sigma$ is a scale parameter, and $\xi$ is the tail shape parameter.

We modeled our circularity data with the distribution of extreme values (Equation (2)) using MATLAB ${ }^{\circ}$, a programming language for technical computing from the MathWorks, Natick, MA, USA. This program has library functions to fit the data to a GEV probability distribution function (pdf) using a Maximum Likelihood Estimation process (mle). The result of the fit is a vector of estimated parameters $\hat{\theta}=(\xi, \sigma, \mu)^{T}$ and a covariance matrix $S_{\hat{\theta}}$ for these parameters.

In order to test the significance of the difference between pdfs, the following analysis was performed (the procedure is explained in two steps in order to describe it clearly):

(1) The Maximum Likelihood Estimation method produces estimators that are asymptotically unbiased following a multivariate Gaussian distribution given by Equation (3) defined as ${ }^{30,31}$

$$
f(\theta)=\frac{1}{\left[(2 \pi)^{3}\left|S_{\hat{\theta}}\right|\right]^{1 / 2}} \exp \left\{-\frac{1}{2}(\theta-\hat{\theta})^{T} S_{\hat{\theta}}^{-1}(\theta-\hat{\theta})\right\}
$$

This equation describes a three-variate probability distribution for the reason that the vector of estimated parameters $\hat{\theta}=(\xi, \sigma, \mu)^{T}$ has three components. It is possible to calculate 
the term inside the exponential, $(\theta-\hat{\theta})^{T} S_{\hat{\theta}}^{-1}(\theta-\hat{\theta})=D^{2}$ in 255 the case in which the covariance matrix $S_{\hat{\theta}}$ is diagonal. Because of the normality of the data, the result is the sum of three independent normal variables with unit variance. Therefore, $D^{2}$ follows a $\chi^{2}$-pdf with $N=3$ degrees of freedom. $^{32}$ This distance, $\mathrm{D}$, can be considered as

260 a deviation of the values of $\theta$ from $\hat{\theta}$, measured in units of the directional standard deviation in the direction of $(\theta-\hat{\theta})$.

(2) The previous fitting procedure was performed for the control group. The result was a vector of distribution fitted parameters and a covariance matrix for those parameters: $\hat{\theta}_{C}=\left(\xi_{C}, \sigma_{C}, \mu_{C}\right)^{T}, S_{\hat{\theta}, C}$.

Then, it was possible to calculate a distance value for deficit groups as:

$$
\begin{aligned}
& D_{C, D 2}^{2}=\left(\hat{\theta}_{D 2}-\hat{\theta}_{C}\right)^{T} S_{\hat{\theta}, C}^{-1}\left(\hat{\theta}_{D 2}-\hat{\theta}_{C}\right) \\
& D_{C, D 8}^{2}=\left(\hat{\theta}_{D 8}-\hat{\theta}_{C}\right)^{T} S_{\hat{\theta}, C}^{-1}\left(\hat{\theta}_{D 8}-\hat{\theta}_{C}\right),
\end{aligned}
$$

and, at the same time, the cumulative probability of the value $\mathrm{D}^{2}$ in both expressions of Equation (4) knowing that they follow a $\chi^{2}$ pdf distribution with 3 degrees of freedom. This probability can be interpreted as a degree of difference among GEV probability distributions of deficit groups and GEVcontrol, that is, how far the probability distribution of deficit groups was from the control group.

\section{Results}

The biometric measurement for the control, D2 and D8 groups consisted of 19 measurements each. Once the zones were selected and the calculations performed, we started the statistical analysis of the results.

The results are shown in Tables 1 and 2. The data tables show statistics about the distribution of the variables in the lens (area and circularity), the eye (area and circularity), and retina (area). Table 1 shows that the average of the estimated area of these structures, except the retina, became smaller with increasing FA deficiency. This also increased the dispersion (standard deviation) among individuals. This is in agreement with the malformations observed in the D2 and D8 groups, with higher severity in D8. Notably, even with 2 weeks of deficit, variability between individuals increased for eye and lens parameters. In the case of the retina, these variations were not as significant. In fact, the area of the retina suffered no
Table 2. Area and circularity (circ) mean values for the skewness and kurtosis estimators of the lens, the eye, and retina from sample groups. C: control group. D2 and D8: FA deficient groups. The symbol $\left(^{*}\right)$ marks the no significant

\begin{tabular}{|c|c|c|c|c|c|c|c|}
\hline \multirow{2}{*}{\multicolumn{2}{|c|}{ Parameter }} & \multicolumn{3}{|c|}{ Skewness } & \multicolumn{3}{|c|}{ Kurtosis } \\
\hline & & $C$ & D2 & D8 & C & D2 & D8 \\
\hline \multirow[t]{2}{*}{ Lens } & Area & -1.11 & 0.64 & 1.03 & 0.92 & -0.17 & $1.38^{*}$ \\
\hline & Circ. & -0.66 & -2.23 & -1.75 & -0.62 & 4.31 & 3.06 \\
\hline \multirow[t]{2}{*}{ Eye } & Area & -0.02 & 0.13 & 0.73 & -1.11 & -1.42 & 1.37 \\
\hline & Circ. & -0.59 & -1.60 & -0.98 & -0.37 & 3.50 & 2.23 \\
\hline Retina & Area & 1.45 & -0.17 & -0.13 & 3.50 & -1.26 & -0.68 \\
\hline
\end{tabular}
differences.

major changes. Increasing the deficit days did not increase the size of the retina.

In the case of circularity parameter, differences in mean values between individuals were not very high, especially in the case of lens circularity (Table 1). This is the reason we calculated higher order statistics (Table 2). The results of the Kruskal-Wallis contrast on the bootstrap estimators of the parameters of Tables 1, 2, and 4 appear in them using the asterisk. This symbol indicates the estimators whose distribution is significantly different from those of the control group. As indicated in the methods section, these cases are those in which the $p$ value is greater than 0.05 .

The role of statistical Skewness (3rd order moment of probability distribution) is to describe the symmetry around the mean. If it is zero, the pdf is symmetric, if not it is asymmetric. The Kurtosis (4th order moment of pdf) measures the degree of sharpness of the pdf peak when reference to a Gaussian distribution, ${ }^{33}$ which is related to the behavior of that distribution in the tails and the existence of outliers. ${ }^{34}$

Lens area skewness showed that the asymmetry value obtained in the control group was negative, indicating that there was some asymmetry between control individuals. In both groups, D2 and D8, different behaviors are observed compared to the control group. The same was observed when analyzing the Kurtosis: the control, D2 and D8 groups appeared clearly different. In case of lens circularity, the skewness changes from negative (control) to positive (D2 and D8). This indicates an increase of asymmetry toward higher values. Since the lens circularity has a maximum of one, this indicates that lens in D2 and D8 increases the circularity respect to control group. The kurtosis changes from negative (control) to positive (D2 and D8). This indicates extended tails of the distribution in case of D2 and D8 revealing the presence of outliers (increase of the probability of large deviation respect to mean and median of the distribution respect to control group, probably due to malformations). The same can be said about eye circularity and area although in this last case, the change to positive kurtosis

Table 1. Area and circularity (circ) mean values for mean and standard deviation bootstrap estimators of the lens, the eye, and retina from sample groups. C: control group. D2 and D8: FA deficient groups. All the differences in distribution of mean and standard deviation are significant $(p<0.05)$.

\begin{tabular}{llccc}
\hline & & \multicolumn{3}{c}{ Mean and Standard Deviation (pixels) } \\
\cline { 3 - 5 } Parameter & & $\mathrm{C}$ & $\mathrm{D} 2$ & $\mathrm{D} 8$ \\
\hline Lens & Area & $285653 \pm 46811$ & $271500 \pm 52489$ & $237862 \pm 97979$ \\
& Circ. & $0.969 \pm 0.016$ & $0.968 \pm 0.035$ & $0.973 \pm 0.019$ \\
Eye & Area & $1175368 \pm 159071$ & $1072368 \pm 201419$ & $1010537 \pm 200348$ \\
& Circ. & $0.960 \pm 0.020$ & $0.971 \pm 0.020$ & $0.966 \pm 0.018$ \\
Retina & Area & $548126 \pm 72675$ & $512521 \pm 115492$ & $520332 \pm 66423$ \\
\hline
\end{tabular}


respect to control group only occurs for D8 group. In the case of the retina, the skewness changes from positive (control) to negative (D2, D8), showing a change in the asymmetry of the distribution. At this point, it is interesting to note that while the mean value of the retina area changes little (Table 1), the change between the control group and D2, D8 is more noticeable in the asymmetry of the distribution. Regarding kurtosis, the change between positive (control) and negative (D2, D8) shows a concentration of the data around the mean and median value in groups D2 and D8 with respect to the control group.

The results of the previous explained fitting procedure to GEV functions (see Section 'Materials and methods') and analysis are presented in Figure 2; we observed that in the D2 and D8 groups, lens and eye circularity pdfs were different from the control pdf. The distance $\mathrm{D}$ between the fitted parameters and the control fitted parameters was very large and the probability of their being different distributions was very high (nearly one), according to the co-variance in the control fitted parameters. In general, the eye and the lens with FA deficiency were more circular. Comparing Tables 1 and 2, it is possible to say that the mean differences between groups when talking about circularity were in the higher order statistics, producing different GEV pdfs.

In this study, we observed that eyes developed in FA deficiency conditions were smaller than those in the control group. So, we calculated the relative decrease $(\Delta)$ compared to the control area with the following formula (Equation (5)). The results are shown in Table 3:

$\begin{aligned} \Delta= & \frac{\text { Control Group_mean Area }- \text { Deficient Group_mean Area }}{\left(\frac{\text { Control Group_mean Area+Deficient Group_mean Area }}{2}\right)} \\ & * 100\end{aligned}$

Table 3 shows that when there was a deficit of FA, the eye became smaller; its size was reduced $9.16 \%$ in the case of D2 and $15.08 \%$ in the case of D8. This reduction was accompanied by a reduction in the size of the lens by $5.05 \%$ in the case of D2 and $18.26 \%$ in the case of D8. Nevertheless, in the case of the retina, the reduction was $6.71 \%$ in $\mathrm{D} 2$, and in the case
Table 3. Values of the relative decrease in ocular structures with FA deficiency, compared to the control group.

\begin{tabular}{lcc}
\hline Structure & D2 (\%) & D8 (\%) \\
\hline Lens & 5.08 & 18.26 \\
Eye & 9.16 & 15.08 \\
Retina & 6.71 & 5.20 \\
\hline
\end{tabular}

of D8, reduction was only $5.20 \%$. Both values are comparable and within the error margins of variability among control group individuals. The data in Table 3 are a rearrangement of those in Table 1, since they express the percentage of variation in the size of the eye, retina, and lens.

With increasing FA deficiency, the eye became increasingly smaller, while the retina did not change its area. For that reason, we hypothesize that the retina must change its geometry in order to fit into a smaller eye. To test this hypothesis, we statistically analyzed the height and the width of the retina (see Figure 3) of the three sample groups and compared them to the results for the control group. The results are shown below, see Table 4 .

The height of the retina decreases in groups D2 and D8 relative to the control while its width increases. The dispersion of the height and width of the retina increases in groups D2 and D8 with respect to the control (greater standard deviation). The degree of asymmetry of the distributions tends to decrease with respect to the control group. In the case of kurtosis, as in the general case of the area of the retina, the width of the retina changes from positive to negative kurtosis, concentrating the values around the mean value.

As shown in Table 4, the height of the retina became smaller when FA deficiency increased. This coincides with the decrease in size of the eye caused by the deficit. But when we analyzed the data, we observed that the retina was wider in the $\mathrm{D} 8$ group.

\section{Discussion}

Lack of FA produces defects in cell growth, mainly in infants whose mothers lacked FA. ${ }^{35} \mathrm{FA}$ is required for DNA, RNA, and protein synthesis and uses two different transport mechanisms to enter mammalian cells: the folate receptor and reduced-folate transporter. ${ }^{36-38}$ Many studies have confirmed the distribution
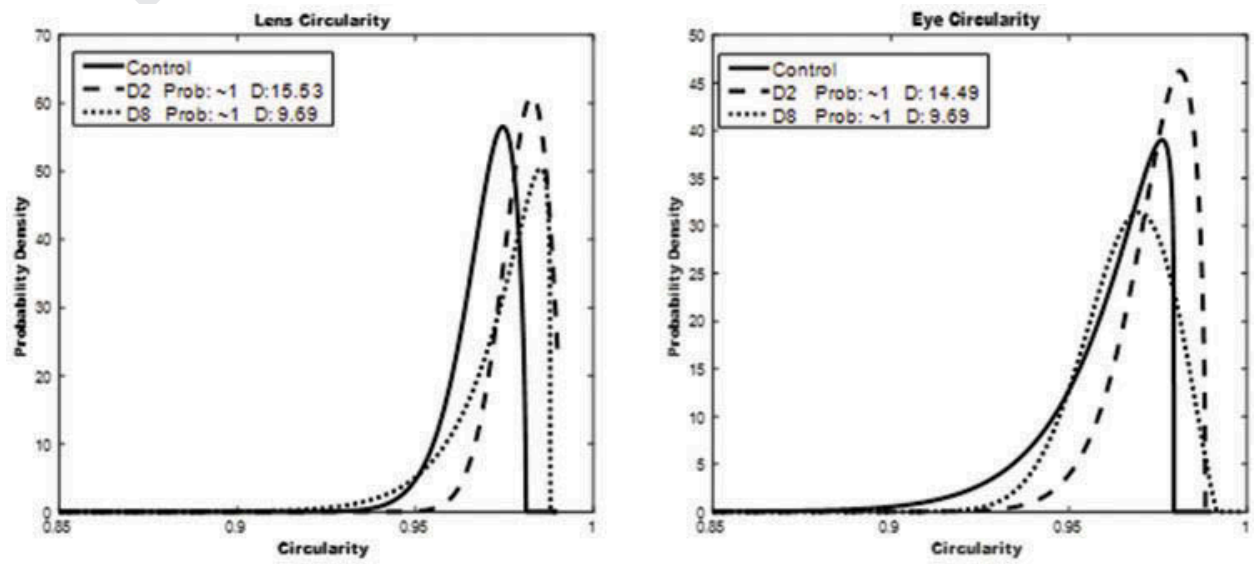

Figure 2. Lens and eye circularity probability distribution for the three sample groups. 


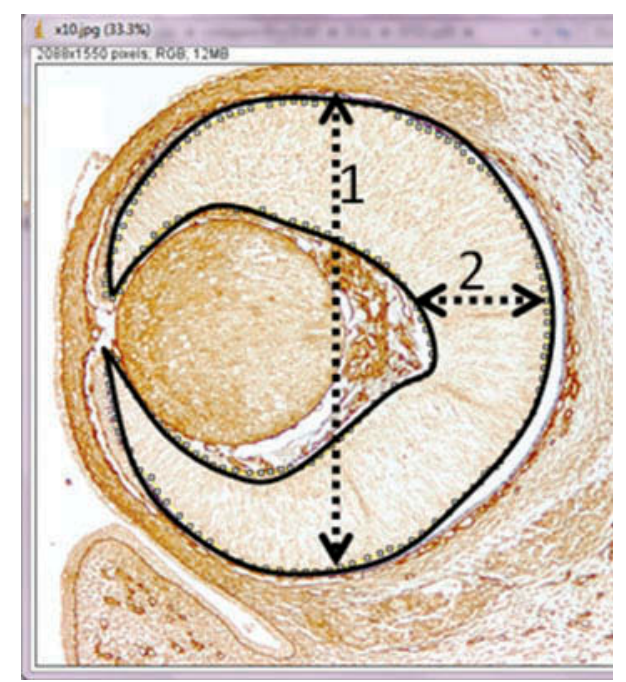

Figure 3. Retinal measurements: Height (arrow 1) and width (arrow 2). Frontal section of E14.5 mouse embryo with $\times 10$ magnification labeled with anti-type IV collagen.

of these two proteins in ocular structures during embryological development. $^{39-42}$

In this study, we are the first to perform ocular biometric measurements in mice embryos from females with FAD diet. For this, in the present article we realize a more comprehensive statistical study to detect significant changes in the biometry of some eye structures. We have shown changes in whole eye and lens sizes even in the group whose mothers were only deprived of FA during the first gestational days. It is known that FA is necessary for normal embryonic cell proliferation processes; deficiency interferes with growth of the embryo. ${ }^{43}$ In fact, low folate intake was associated with a twofold increase in the risk of low infant birth weights. ${ }^{44}$ Certainly, in our deficient embryos, the size of the

410 whole eye and lens was always smaller than that of controls possibly due to reduced cell proliferation. Some studies have demonstrated that FA deficiency decreased cell proliferation. ${ }^{21,45}$

Strangely enough, comparing neural retinas, we have shown that despite of the deficit, the area size did not change, not even when the deficit was very severe as in D8. To confirm this, we measured height and width of the retina, which revealed that in the D8 group, the neural retina was thicker than control embryos. Likewise, the increase in the size of the neural retina has been seen in various studies. ${ }^{46,47}$ Experimental studies ${ }^{48,49}$ have revealed that reduced central nervous system apoptosis results in multiple hyperplasias, ectopic cell masses, disorganized cellular structures, and excessive numbers of neurons in the cortex, cerebellum, and retina. It has been known for a long time that the removal of cells by programmed cell death is a mechanism of development. cells are generated during the development of a number of

systems, such as the central nervous system ${ }^{50}$ including the retina. ${ }^{51}$ Changes in the amount and composition of extracellular matrix components induce modifications in the apoptosis/survival cellular. $^{52,53}$ Moreover, it has been shown that maternal dietary choline deficiency alters the retinal development and structural organization in the offspring of mouse dams. ${ }^{54}$ Previously, it has been reported that folate and choline metabolism are interrelated, and low levels of FA produce a decrease in choline status ${ }^{55-57}$ affecting the development of the nervous system ${ }^{58}$ and possibly ocular development.

Regarding circularity measures, we have observed that eyes and lenses of embryos from maternal FAD diet were more circular than control. In brief, our results show an association between maternal FAD diet and variations in ocular biometric parameters: area and circularity.

On the other hand, type IV collagen forms an integrated network within the laminin scaffold providing strength and stability. ${ }^{59}$ These proteins are important as structural components of the native basement membranes and subsequently as matrices supporting cell adhesion, migration, and proliferation. ${ }^{14,60}$ It is not surprising that any disruption in basement membrane proteins such as type IV collagen and/or laminin-1 would result in changes or alterations in tissue morphology. In our previous work, ${ }^{13}$ we have shown that FAD diet produces changes in the expression of both proteins in the lens tissue, this is probably one of the causes of lens shape alteration and its decrease in size. It also seems logical to assume that changes observed in the eye and retina could be due to the alteration of extracellular matrix proteins.

An adequate contribution of FA in the diet is not only essential during embryonic development for the correct formation of the nervous system but also required for the growth, maturation, and correct functioning of the nervous system in children and the elderly. ${ }^{61}$ In elderly, FAD maybe due not only to low intake of FA but also to other circumstances (bad absorption, alcoholism, medication, etc.). Optic neuropathy ${ }^{62-64}$ and macular degeneration ${ }^{65,66}$ are related to FAD.

Most pregnancies in humans are unplanned. On average, the first antenatal visit is 9 weeks. ${ }^{67}$ However, the human eye begins to develop by 7 weeks of fetal life. So, pregnancy can begin with maternal FAD. Due to the great susceptibility of ocular development in the absence of FA, as we have shown in our results, babies with poor visual function and without other alteration, it could be suspected that it was due to a small maternal deficit of FA at the beginning of pregnancy.

\section{Conclusions}

According to our results, it is clear that FA deficiency produces morphological changes in lens, retina, and entire eye. These changes were also noticeable in embryos obtained from mothers

Table 4. Statistical bootstrap estimator mean values of the height and width of the retina for sample groups. C: control group. D2 and D8: FA deficient groups. Estimators: mean, std. deviation, skewness, kurtosis. All the differences from the control group are significant $(p<0.05)$.

\begin{tabular}{lcccccc}
\hline & \multicolumn{3}{c}{ Height of the retina (pixels) } & & \multicolumn{3}{c}{ Width of the retina (pixels) } \\
\cline { 2 - 4 } & Control & D2 & D8 & & Control & D2 \\
\hline Mean & 1150.58 & 1097.11 & 1041.47 & 321.32 & 301.58 & 337.00 \\
Std. Deviation & 98.340 & 139.616 & 136.708 & 20.502 & 367 & 35.873 \\
Skewness & 0.208 & 0.093 & -0.053 & 0.775 & -0.481 & -0.107 \\
Kurtosis & -0.720 & -1.213 & 0.082 & 0.403 & -0.257 & -0.436 \\
\hline
\end{tabular}


subjected to FA-deficient diet only 2 weeks from mating ( equivalent to 7 weeks in humans). In summary, the severe changes in eye structures biometry observed in our FA-deficient mice embryos revealed the important role of FA during embryonic development. It is evident that FA plays a significant role in extracellular matrix protein synthesis and FA deficiency contributes to many kinds of ocular disorders. Changes introduced by FA deficiency were visible in the short term (2 weeks) and clearly evident in the long term (8 weeks) in mice. As a final conclusion, perhaps some visual anomalies are related to deficient states of FA during the first gestational weeks in women.

\section{Acknowledgments}

The authors thank Alda Serrano Javier from Optics Department for reviewing the draft of the paper and for his helpful suggestions and comments on this manuscript. The authors would also like to acknowledge Dolores Arroyo and Alicia Cerro from the Anatomy and Human Embryology Department for their excellent technical assistance.

\section{Disclosure statement}

No conflicts of interest, financial or otherwise, are declared by the authors.

\section{$495 \quad$ Funding}

This study was supported by grants to the Complutense Research Group 920202 from the Spanish Ministerio de Sanidad, Servicios Sociales E Igualdad (PI06/0184 and PS09/01762). Likewise, it was supported by grants from the Spanish Ministerio de Economía y Competitividad (TEC2013-40442). Finally, this article was done with a pre-doctoral fellowship awarded by Banco Santander/Universidad Complutense de Madrid (CT27/16 - CT28/16).

\section{ORCID}

Ouafa Sijilmassi (D) http://orcid.org/0000-0003-0513-0229

\section{References}

1. Hoar RM. Embryology of the eye. Environ. Health Perspect. 1982;44:31-34. doi:10.1289/ehp.824431.

2. Pei Y, Rhodin J. The prenatal development of the mouse eye. Anat Rec. 1970;168(1):105-25. doi:10.1002/ar.1091680109.

3. Cvekl A, Tamm ER. Anterior eye development and ocular mesenchyme: new insights from mouse models and human diseases. Bioessays. 2004;26(4):374-86. doi:10.1002/bies.20009.

4. Tang LS, Santillano DR, Wlodarczyk BJ, Miranda RC, Finnell RH. Role of folbp1 in the regional regulation of apoptosis and cell proliferation in the developing neural tube and craniofacies. AM J MED GENET C. 2005;135C(1). doi:10.1002/ajmg.c.30053.

5. Maddox DM, Manlapat A, Roon P, Prasad P, Ganapathy V, Smith SB. Reduced-folate carrier (RFC) is expressed in placenta and yolk sac, as well as in cells of the developing forebrain, hindbrain, neural tube, craniofacial region, eye, limb buds and heart. BMC Dev Biol. 2003;3(1):6. doi:10.1186/1471-213X-3-6.

6. Van Der Put NM, Van Straaten HW, Trijbels FJ, Blom HJ. Folate, homocysteine and neural tube defects: an overview. Exp Biol Med. 2001;226(4):243-70. doi:10.1177/153537020122600402.

7. Copp AJ, Greene ND. Neural tube defects-disorders of neurulation and related embryonic processes. WIRES Dev Biol. 2013;2 (2):213-27. doi:10.1002/wdev.71.
8. Blom HJ, Shaw GM, Den Heijer M, Finnell RH. Neural tube defects and folate: case far from closed. Nat Rev Neurosci. 2006;7(9):724-31. doi:10.1038/nrn1986.

9. Armstrong RC, Monie I. Congenital eye defects in rats following maternal folic-acid deficiency during pregnancy. Development. 1966;16:531-42.

10. Maestro-de-las-Casas C, Pérez-Miguelsanz J, LópezGordillo Y, Maldonado E, Partearroyo T, Varela-Moreiras G, Martínez-Álvarez C. Maternal folic acid-deficient diet causes congenital malformations in the mouse eye. Birth Defects Res A Clin Mol Teratol. 2013;97(9):587-96. doi:10.1002/ bdra.23176.

11. Shaw GM, Carmichael SL, Laurent C, Louik C, Finnell RH, Lammer EJ. Nutrient intakes in women and risks of anophthalmia and microphthalmia in their offspring. Birth Defects Res A Clin Mol Teratol. 2007;79(10):708-13. doi:10.1002/bdra.20398.

12. Weber KA, Yang W, Carmichael SL, Shaw GM, Study NBDP. Nutrient intake in women before conception and risks of anophthalmia and microphthalmia in their offspring. Birth Defects Res. 2018. doi:10.1002/bdr2.1201.

13. Sijilmassi O, López Alonso J, Barrio Asensio M, Del Río Sevilla A. Collagen IV and laminin-1 expression in embryonic mouse lens using principal components analysis technique. J Microsc. 2018. doi:10.1111/jmi.12709.

14. Rozario T, DeSimone DW. The extracellular matrix in development and morphogenesis: a dynamic view. Dev Biol. 2010;341 (1):126-40. doi:10.1016/j.ydbio.2009.10.026.

15. Rasband W ImageJ, US national institutes of health. Bethesda (Maryland, USA);2011. http://imagej.nih.gov/ij/.

16. Toolbox MNN. version 8.3. 0 (R2014a). Vol. 4. Natick (Massachusetts): The MathWorks Inc; 2014.

17. Walzem RL, Clifford AJ. Folate deficiency in rats fed diets containing free amino acids or intact proteins. J Nutr. 1988;118 (9):1089-96. doi:10.1093/jn/118.9.1089.

18. COUNCIL NR. COMMITTEE ON ANIMAL NUTRITION. SUBCOMMITTEE ON POULTRY NUTRITION. Nutrient requirements of poultry. Washington (DC): National Academy Press; 1995.

19. Burgoon JM, Selhub J, Nadeau M, Sadler TW. Investigation of the effects of folate deficiency on embryonic development through the establishment of a folate deficient mouse model. Teratology. 2002;65(5):219-27. doi:10.1002/tera.10040.

20. Burren KA, Savery D, Massa V, Kok RM, Scott JM, Blom HJ, Copp AJ, Greene ND. Gene-environment interactions in the causation of neural tube defects: folate deficiency increases susceptibility conferred by loss of Pax3 function. Hum Mol Genet. 2008;17(23):3675-85. doi:10.1093/hmg/ddn262.

21. Maldonado E, Murillo J, Barrio C, Del Rio A, Perez-Miguelsanz J, Lopez-Gordillo Y, Partearroyo T, Paradas I, Maestro C, MartínezSanz E, et al. Occurrence of cleft-palate and alteration of Tgf-beta (3) expression and the mechanisms leading to palatal fusion in mice following dietary folic-acid deficiency. Cells Tissues Organs. 2011;194(5):406-20. doi:10.1159/000323213.

22. Otis EM, Brent R. Equivalent ages in mouse and human embryos. Anat Rec. 1954;120(1):33-63. doi:10.1002/ar.1091200104.

23. Martínez-Sanz E, Del Río A, Barrio C, Murillo J, Maldonado E, Garcillán B, Amorós M, Fuerte T, Fernández A, Trinidad E, et al. Alteration of medial-edge epithelium cell adhesion in two Tgf- $\beta 3$ null mouse strains. Differentiation. 2008;76(4):417-30. doi:10.1111/j.1432-0436.2007.00226.x.

24. IBM_Corp R. IBM SPSS statistics for windows. Armonk (New Yourk (NY)): IBM Corp; 2010.

25. Chernick MR. Bootstrap methods: a guide for practitioners and researchers. Hoboken (New Jersey): John Wiley \& Sons. Inc.; 2008.

26. Goodhue DL, Lewis W, Thompson R. Does PLS have advantages for small sample size or non-normal data? Mis Quarterly. 2012;36 (3):981-1001. doi:10.2307/41703490.

27. Markose S, Alentorn A. The generalized extreme value distribution, implied tail index, and option pricing. J Derivatives. 2011;18 (3):35-60. doi:10.3905/jod.2011.18.3.035. 
28. Bali TG. The generalized extreme value distribution. Econ Lett. 2003;79(3):423-27. doi:10.1016/S0165-1765(03)00035-1.

29. Conniffe D. The generalised extreme value distribution as utility function. Econ Soc Rev. 2007;38:275.

30. Peña D. Fundamentos de estadística. Madrid: Alianza; 2008.

31. Edwards A, Likelihood F. Expanded edition. Baltimore: Johns Hopkins Univ. Press; 1992.

32. El Adlouni S, Ouarda T, Zhang X, Roy R, Bobée B. Generalized maximum likelihood estimators for the nonstationary generalized extreme value model. Water Resour Res. 2007;43:3. doi:10.1029/ 2005WR004545.

33. MacGillivray H, Balanda K. The relationships between skewness and kurtosis. Aust N Z J Stat. 1988;30(3):319-37. doi:10.1111/ j.1467-842X.1988.tb00626.x.

34. Westfall PH. Kurtosis as peakedness, 1905-2014. RIP. Am. Stat. 2014;68(3):191-95. doi:10.1080/00031305.2014.917055.

35. Threlfall G. Cell proliferation in the rat kidney induced by folic acid. Cell Prolif. 1968;1(4):383-92. doi:10.1111/j.1365-2184.1968. tb00967.x.

36. Sirotnak F, Tolner B. Carrier-mediated membrane transport of folates in mammalian cells. Annu Rev Nutr. 1999;19(1):91-122. doi:10.1146/annurev.nutr.19.1.91.

620 37. Antony AC. Folate receptors. Annu Rev Nutr. 1996;16(1):501-21. doi:10.1146/annurev.nu.16.070196.002441.

38. Rajgopal A, Sierra EE, Zhao RB, Goldman ID. Expression of the reduced folate carrier SLC19A1 in IEC-6 cells results in two distinct transport activities. Am J Physiol Cell Physiol. 2001;281 (5):C1579-C86. doi:10.1152/ajpcell.2001.281.5.C1579.

39. Saitsu H, Ishibashi M, Nakano H, Shiota K. Spatial and temporal expression of folate-binding protein 1 (Fbp 1$)$ is closely associated with anterior neural tube closure in mice. Dev Dyn. 2003;226 (1):112-17. doi:10.1002/dvdy.10203.

630 40. Bozard BR, Ganapathy PS, Duplantier J, Mysona B, Ha Y, Roon P, Smith R, Goldman ID, Prasad P, Martin P, et al. Molecular and biochemical characterization of folate transport proteins in retinal Muller cells. Invest Ophthalmol Visual Sci. 2010;51(6):3226-35. doi:10.1167/iovs.09-4833.

635 41. Bridges CC, El-Sherbeny A, Ola MS, Ganapathy V, Smith SB. Transcellular transfer of folate across the retinal pigment epithelium. Curr Eye Res. 2002;24(2):129-38. doi:10.1076/ceyr.24.2.129.8167.

42. Dun Y, Prasad P, Mysona B, Van Ells T, Ganapathy V, Smith S. Analysis of folate receptor (FR) alpha in ganglion and Müller cells and FR delta in RPE. Invest Ophthalmol Visual Sci. 2005;46:2969.

43. Luke B. Nutrition during pregnancy: part I, weight gain: part II, nutrient supplements. JAMA. 265(2):281-82. doi:10.1001/ jama.1991.03460020139044.

44. Scholl TO, Hediger ML, Schall JI, Khoo CS, Fischer RL. Dietary and serum folate: their influence on the outcome of pregnancy. Am J Clin Nutr. 1996;63(4):520-25. doi:10.1093/ajcn/63.4.520.

45. Moussa C, Ross N, Jolette P, MacFarlane AJ. Altered folate metabolism modifies cell proliferation and progesterone secretion in human placental choriocarcinoma JEG-3 cells. Br J Nutr. 2015;114 (6):844-52. doi: $10.1017 /$ S0007114515002688.

46. Dunker N, Krieglstein K. Reduced programmed cell death in the retina and defects in lens and cornea of Tgf beta 2(-/-)Tgf beta 3 (-/-) double-deficient mice. Cell Tissue Res. 2003;313(1):1-10. doi:10.1007/s00441-003-0761-x.

655 47. Cecconi F, Alvarez-Bolado G, Meyer BI, Roth KA, Gruss P. Apaf1 (CED-4 homolog) regulates programmed cell death in mammalian development. Cell. 1998;94(6):727-37. doi:10.1016/S00928674(00)81732-8.

48. Oppenheim RW. Cell-death during development of the nervous-system. Annu Rev Neurosci. 1991;14:453-501.
49. Kuida K, Zheng TS, Na SQ, Kuan CY, Yang D, Karasuyama H, Rakic P, Flavell R. Decreased apoptosis in the brain and premature lethality in CPP32-deficient mice. Nature. 1996;384 (6607):368-72. doi:10.1038/384368a0.

50. Conradt B. Genetic control of programmed cell death during animal development. Annu Rev Genet. 2009;43:493-523. doi:10.1146/annurev.genet.42.110807.091533.

51. Laemle LK, Puszkarczuk M, Feinberg RN. Apoptosis in early ocular morphogenesis in the mouse. Dev Brain Res. 1999;112 (1):129-33. doi:10.1016/S0165-3806(98)00153-9.

52. Farrelly N, Lee Y-J, Oliver J, Dive C, Streuli CH. Extracellular matrix regulates apoptosis in mammary epithelium through a control on insulin signaling. J Cell Biol. 1999;144(6):1337-48. doi:10.1083/jcb.144.6.1337.

53. Frisch SM, Francis H. Disruption of epithelial cell-matrix interactions induces apoptosis. J Cell Biol. 1994;124(4):619-26. doi:10.1083/jcb.124.4.619.

54. Surzenko N, Trujillo-González I, Zeisel SH. Low intake of choline during pregnancy leads to aberrant retinal architecture and poor visual function in the offspring. FASEB J. 2016;30:679-9.

55. Varela-Moreiras G, Selhub J, Da Costa K, Zeisel SH. Effect of chronic choline deficiency in rats on liver folate content and distribution. J. Nutr. Biochem. 1992;3(10):519-22. doi:10.1016/ 0955-2863(92)90073-R.

56. Kim YI, Miller JW, Da Costa KA, Nadeau M, Smith D, Selhub J, Zeisel SH, Mason JB. Folate deficiency causes secondary depletion of choline and phosphocholine in rat liver. J. Nutr. 1994;124 (11):2197-203. doi:10.1093/jn/124.11.2197.

57. Niculescu MD, Zeisel SH, Diet M. Donors and DNA methylation: interactions between dietary folate, methionine and choline. J. Nutr. 2002;132(8):2333S-2335S. doi:10.1093/jn/132.8.2333S.

58. Craciunescu CN, Johnson AR, Zeisel SH. Dietary choline reverses some, but not all, effects of folate deficiency on neurogenesis and apoptosis in fetal mouse brain. J Nutr. 2010;140(6):1162-66. doi:10.3945/jn.110.122044.

59. Danysh BP, Duncan MK. The lens capsule. Exp Eye Res. 2009;88 (2):151-64. doi:10.1016/j.exer.2008.08.002.

60. Olivero DK, Furcht LT. Type-IV collagen, laminin, and fibronectin promote the adhesion and migration of rabbit lens epithelial-cells in-vitro. Invest Ophthalmol Visual Sci. 1993;34:2825-34.

61. Reynolds E. Vitamin B12, folic acid, and the nervous system. Lancet Neurol. 2006;5(11):949-60. doi:10.1016/S1474-4422(06) 70598-1.

62. Golnik KC, Schaible ER. Folate-responsive optic neuropathy. J Neuroophthalmology. 1994;14(3):163-69. doi:10.1097/ 00041327-199409000-00008.

63. Hsu CT, Miller NR, Wray ML. Optic neuropathy from folic acid deficiency without alcohol abuse. Ophthalmologica. 2002;216 (1):65-67. doi:10.1159/000048300.

64. De Silva P, Jayamanne G, Bolton R. Folic acid deficiency optic neuropathy: a case report. J Med Case Rep. 2008;2(1):299. doi:10.1186/1752-1947-2-299.

65. Huang P, Wang F, Sah BK, Jiang J, Ni Z, Wang J, Sun X. Homocysteine and the risk of age-related macular degeneration: a systematic review and meta-analysis. Sci Rep. 2015;5:10585. doi:10.1038/srep10585.

66. Pinna A, Zaccheddu F, Boscia F, Carru C, Solinas G. Homocysteine and risk of age-related macular degeneration: a systematic review and meta-analysis. Acta Ophthalmol. 2018;96(3):e269-e276. doi:10.1111/aos.13343.

67. Herrmann W, Obeid R. The mandatory fortification of staple foods with folic acid: a current controversy in Germany. DTSCH ARZTEBL INT. 2011;108(15):249-54. doi:10.3238/arztebl.2011.0249. doi:10.1146/annurev.ne.14.030191.002321. 